\title{
A New Fuzzy DEA/AHP Methodology for the Facility Layout Problem in a Fuzzy Environment
}

\author{
C.-K. Hu${ }^{1}$, F.-B. $\mathrm{Liu}^{2}$, C.-F. $\mathrm{Hu}^{3}$ \\ ${ }^{1}$ Department of International Business, Kao Yuan University \\ No. 1821, Jhongshan Rd., Lujhu, Kaohsiung, Taiwan \\ t80147@cc.kyu.edu.tw \\ ${ }^{2}$ Department of Mechanical and Automation Engineering, I-Shou University \\ No.1, Sec. 1, Syuecheng Rd., Dashu District, Kaohsiung, Taiwan \\ fliu@isu.edu.tw \\ ${ }^{3}$ Department of Applied Mathematics, National Chiayi University \\ No. 300 Syuefu Rd., Chiayi, Taiwan \\ cfhu@mail.ncyu.edu.tw
}

\section{Extended Abstract}

The facility layout problem has been a subject of tremendous interest in manufacturing system. It is concerned with the allocation of activities to space such that a set of criteria is met and/or some objectives are optimized. A number of formulations have been developed for the facility layout problem. Most of them involve over simplifying assumptions and request overwhelming computational efforts such that they cannot be manipulated with ease in practice [1]. To cope with the difficulties being the cause of this complexity and the vagueness involved in the real-world decision-making problem, this work develops a novel approach combining the Tchebycheff model in fuzzy data envelopment analysis (DEA) and the analytical hierarchical process (AHP) for evaluating the facility layout problem with multiple fuzzy criteria.

The hybrid fuzzy DEA/AHP approach takes the best of both fuzzy DEA and AHP methods by avoiding the pitfalls of each. It consists of two stages for ranking DMUs with multiple fuzzy criteria. In the first stage of the ranking method, Tchebycheff model in DEA with multiple fuzzy criteria is employed to construct the pairwise comparison matrix. Thus, there is no subjective assessment of a decision-maker evaluation involved. In the second stage of the ranking method, a single level AHP is utilized to fully rank units according to the pairwise evaluation matrix generated in the first stage, which leads to a high discrimination of the decision making units. It shows that the proposed method presents a logical ranking of DMUs that is compatible with the efficient/inefficient classification derived from fuzzy DEA.

An empirical study on the facility layout designs of the plastic profile production system is provided to illustrate the superiority of the proposed approach. In our empirical study, fuzzy criteria, for example, flexibility in volume and variety and quality related to the product and production, as well as quantitative criteria such as material handling cost, adjacency score, shape ratio, and material handling vehicle utilization in the decision process are considered. To take care of the multiple criteria by fuzzy DEA, the criteria that are to be minimized are viewed as inputs and the criteria to be maximized are considered as outputs. Eighteen facility layout alternatives are evaluated using four output variables and two input variables. Table 1 shows the results of ranking by the fuzzy DEA model, the method in [1] and the proposed ranking method by the hybrid fuzzy DEA/AHP. The numbers in parentheses are rankings of the corresponding DMUs. According to table 1, the proposed hybrid fuzzy DEA/AHP ranking method leads to more discrimination. In addition, from Table 1, DMUs which are efficient in the fuzzy DEA, rank higher than inefficient DMUs by using the proposed ranking method. It shows the ranking by the proposed approach is compatible with the result by fuzzy DEA and it furthers the analysis by providing fully ranking for all units with multiple fuzzy criteria 
Table 1: Ranking by different methods.

\begin{tabular}{crrr}
\hline DMUs & Fuzzy DEA & Method in $[22]$ & $\begin{array}{r}\text { weight vector in } \\
\text { Fuzzy DEA/AHP }\end{array}$ \\
\hline 1 & $0.9595(14)$ & $0.949(14)$ & $0.2419804(13)$ \\
2 & $1.0000(1)$ & $0.988(11)$ & $0.2422439(6)$ \\
3 & $1.0000(1)$ & $0.997(10)$ & $0.2422704(4)$ \\
4 & $0.9098(15)$ & $0.985(12)$ & $0.2350762(14)$ \\
5 & $1.0000(1)$ & $1.000(1)$ & $0.2424019(1)$ \\
6 & $1.0000(1)$ & $0.973(13)$ & $0.2422452(5)$ \\
7 & $1.0000(1)$ & $1.000(1)$ & $0.2421172(10)$ \\
8 & $0.8617(16)$ & $0.852(17)$ & $0.1945052(18)$ \\
9 & $0.9989(13)$ & $0.889(15)$ & $0.2250112(15)$ \\
10 & $1.0000(1)$ & $1.000(1)$ & $0.2423731(2)$ \\
11 & $1.0000(1)$ & $0.998(9)$ & $0.2421616(7)$ \\
12 & $1.0000(1)$ & $1.000(1)$ & $0.2421571(8)$ \\
13 & $0.7901(18)$ & $0.776(18)$ & $0.2190128(16)$ \\
14 & $1.0000(1)$ & $1.000(1)$ & $0.2420896(12)$ \\
15 & $1.0000(1)$ & $1.000(1)$ & $0.2422820(3)$ \\
16 & $1.0000(1)$ & $1.000(1)$ & $0.2421566(9)$ \\
17 & $1.0000(1)$ & $1.000(1)$ & $0.2420933(11)$ \\
18 & $0.8611(17)$ & $0.857(16)$ & $0.2138516(17)$ \\
\hline
\end{tabular}

\section{References}

[1] T. Ertay, D. Ruan, U. R. Tuzkaya, "Integrating data envelopment analysis and analytic hierarchy for the facility layout design in manufacturing systems," Inf. Sci., vol. 176, pp. 237-262, 2006. 